\title{
Estudo in vitro do efeito do tempo de fotopolimerização na pigmentação de resinas compostas
}

In vitro study of the effect of the photopolymerization time on the pigmentation of composite resins Estudio in vitro del efecto del tiempo de fotopolimerización en la pigmentación de resinas compuestas

\author{
Luiz Henrique Soares TORRES \\ Luiza Monzoli COVRE ${ }^{2}$
}

Caio Pimenteira UCHÔA ${ }^{3}$

Déborah Laurindo Pereira SANTOS ${ }^{1}$

Renato Torres AUGUSTO NETO ${ }^{1}$

Anuar Antônio XIBLE ${ }^{4}$

Valfrido Antônio PEREIRA FILHO ${ }^{1}$

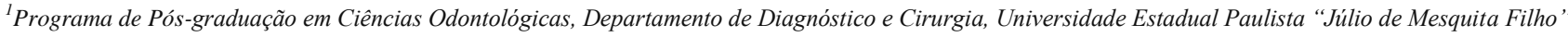 \\ (UNESP) Faculdade de Odontologia de Araraquara, 14801-903, Araraquara-SP, Brasil \\ ${ }_{2}^{2}$ Programa de Pós-graduação em Clínica Integrada, Universidade Estadual Paulista "Júlio de Mesquita Filho" (UNESP) Faculdade de Odontologia de Araçatuba, \\ 16066-840, Araçatuba-SP, Brasil \\ ${ }^{3}$ Departamento de Cirurgia e Traumatologia Bucomaxilofacial, Hospital Universitário Oswaldo Cruz, UPE Universidade de Pernambuco, $50100-130$ Recife-PE, Brasil \\ ${ }^{4}$ Departamento de Prótese Dentária, Universidade Federal do Espírito Santo - UFES, 29047-105 Vitória-ES, Brasil
}

\section{Resumo}

As resinas compostas fotopolimerizáveis têm tido cada vez mais indicações na odontologia restauradora devido às vantagens que apresentam. Porém variações na técnica de fotoativação, densidade de energia, intensidade da luz e tempo de irradiação podem gerar polimerização incompleta, o que pode resultar em uma resina composta com alta porosidade, pouca dureza, baixo polimento, alta capacidade de pigmentação ou efeitos tóxicos na polpa. Este estudo tem por objetivo analisar o efeito do tempo de polimerização na pigmentação extrínseca de três resinas compostas fotopolimerizáveis. Utilizando uma fonte de LED, foram confeccionados 180 espécimes de resina composta microhíbrida, nanohíbrida e nanoparticulada, com diferentes protocolos de polimerização. Os espécimes foram subdivididos em grupos de acordo com o agente pigmentante. Com o uso de um colorímetro, foram analisados quanto à mudança de cor ( $\Delta \mathrm{E})$. Os dados foram submetidos ao teste ANOVA e Duncan $(\alpha=0,05)$. A resina composta microhíbrida apresentou os menores valores de $\Delta \mathrm{E}$ em comparação à nanoparticulada e à nanohíbrida quando imersos em vinho. Quanto maior o tempo de polimerização menores valores de $\Delta \mathrm{E}$ foram obtidos. Não somente o tempo de polimerização influencia no grau de pigmentação, mas também fatores intrínsecos às resinas compostas tais como carga, agente fotoiniciador e matriz orgânica.

Descritores: Autocura de Resinas Dentárias; Materiais Dentários; Pigmentação.

\section{Abstract}

The composite resins have been widely recommended in restorative dentistry due the advantages offered. Although variations in the energy density, light intensity, irradiation time and curing technique can lead to incomplete polymerization, which may result in a resin with high porosity, low hardness, low polishing, high staining capacity or toxic effects in the pulp. This study aims to analyze the effect of setting time in the extrinsic pigmentation three light-cured composite resins. 180 specimens were prepared of microhybrid, nanohybrid and nanoparticle composite resin with different polymerization protocols. The specimens were subdivided into groups according to the size of the inorganic filler and staining agent. Subsequently they were analyzed for color change (according to CIElab code). The data were submitted to ANOVA and Duncan. The microhybrid composite resin showed the lowest $\Delta \mathrm{E}$ values when compared to the nanoparticle and nanohybrid when immersed in wine. As the polymerization time was increased afforded lower $\Delta \mathrm{E}$ values. Not only the curing time influences the degree of pigmentation, but also intrinsic factors to composites as a filler, photoinitiator agent and organic matrix.

Descriptors: Self-Curing of Dental Resins; Dental Materials, Pigmentation.

\section{Resumen}

Las resinas compuestas fotopolimerizables han tenido cada vez más indicaciones en la odontología restauradora debido a las ventajas que presentan. Sin embargo, variaciones en la técnica de fotoactivación, densidad de energía, intensidad de la luz y tiempo de irradiación pueden generar una polimerización incompleta, lo que puede resultar en una resina compuesta con alta porosidad, poca dureza, bajo pulido, alta capacidad de pigmentación o efectos tóxicos en la pulpa. Este estudio tiene por objetivo analizar el efecto del tiempo de polimerización en la pigmentación extrínseca de tres resinas compuestas fotopolimerizables. Utilizando una fuente de LED, fueron confeccionados 180 especímenes de resina compuesta microhíbrida, nanohíbrida y nanoparticulada, con diferentes protocolos de polimerización. Los especímenes se dividieron en grupos de acuerdo con el agente pigmentante. Con el uso de un colorímetro, se analizaron el cambio de color $(\Delta \mathrm{E})$. Los datos fueron sometidos a la prueba ANOVA y Duncan $(\alpha=0,05)$. La resina compuesta microhíbrida presentó los menores valores de $\Delta \mathrm{E}$ en comparación con la nanoparticulada y la nanohíbrida al sumergirse en vino. Cuanto mayor el tiempo de polimerización menores valores de $\Delta \mathrm{E}$ fueron obtenidos. No sólo el tiempo de polimerización influye en el grado de pigmentación, sino también factores intrínsecos a las resinas compuestas como carga, agente fotoiniciador y matriz orgánica.

Descriptores: Autocura de Resinas Dentales; Materiales Dentales; Pigmentación.

\section{INTRODUÇÃO}

A Odontologia tem avançado muito nas últimas décadas, principalmente na área restauradora com o aprimoramento das resinas compostas (RC), sistemas adesivos e materiais restauradores indiretos à base de porcelanas ${ }^{1}$. A RC é amplamente usada como material restaurador direto na prática atual pelo potencial estético e facilidade de manipulação ${ }^{2}$. Entretanto ainda apresenta problemas tais como as mudanças de cor ao longo do tempo de uso clínico, o que pode levar a trocas de restaurações e, consequentemente, aumento de custos para os pacientes e desgaste do dente ${ }^{3}$.
Diversos estudos sobre a estabilidade de cor das RC fotoativadas têm mostrado que bebidas como café, chá, vinho tinto e cola, bem como enxaguantes bucais, podem causar sua pigmentação em diferentes graus. O potencial de coloração destas bebidas e soluções varia de acordo com a composição e propriedades das RC. A polimerização é um dos fatores que influencia no sucesso das restaurações ${ }^{4}$. Cebalhos $^{5}$ e Rahiotis ${ }^{6}$ sugerem que a polimerização incompleta pode resultar em uma RC com alta porosidade, pouca dureza, baixo polimento, alta capacidade de pigmentação e até mesmo efeitos 
tóxicos na polpa causada pelos monômeros livres ${ }^{7-8}$.

A pigmentação pode ser causada pela porosidade das restaurações aliada ao processo de absorção/adsorção de fluidos orais no material; ainda mais, a degradação da superfície pode se tornar acelerada favorecendo a pigmentação extrínseca ${ }^{9}$. Dentre os fatores que influenciam na absorção de pigmentos pelas resinas compostas, pode-se citar o grau de conversão de monômeros em polímeros10 que é influenciado não só pela intensidade e comprimento de onda de luz emitido pelos aparelhos de fotopolimerização usados para excitar as moléculas do fotoiniciador, mas também pelo tempo de irradiação e da composição do material (tipo de matriz resinosa e ao tamanho das partículas de carga) ${ }^{4,11,12}$. Além disso as características de cura de uma RC são altamente dependentes do tipo e quantidade de fotoiniciadores presentes ${ }^{13}$. A canforoquinona (CQ) é o agente fotoiniciador utilizado na maioria das marcas comerciais disponíveis no mercado. Entretanto, o amarelamento do compósito devido à sua incorporação e a necessidade do uso de uma amina terciária pode causar a descoloração futura do material. Para contornar este problema, alguns fabricantes diminuem a quantidade de CQ ou utilizam outros fotoiniciadores especificamente em cores mais claras de resinas compostas ${ }^{14}$.

Dentre outros fotoiniciadores que foram propostos para uso em RC além da CQ, há o PPD (1fenil1,2-propanodiona), o Lucirin TPO ou MAPO (óxido mono-acil-fosfínico) e o Irgacure 819 ou BAPO (óxido bis-acil fosfínico). Estes fotoiniciadores possuem uma cor mais clara que a CQ, interferindo de maneira menos significativamente na cor final dos compósitos ${ }^{15}$. Entretanto, Asmussen, Peutzfeldt ${ }^{16}$ observaram que a polimerização das RC que contém PPD acontece de forma mais lenta que a CQ. Isto sugere que o uso de RC contendo PPD é favorável para prevenir a formação de gaps durante a fotopolimerização, porém necessitam de maior tempo de irradiação. Assim, três principais fatores são necessários para a correta fotopolimerização: o correto comprimento de onda da luz, tempo de cura e intensidade de luz suficiente ${ }^{12,17}$.

Considerando os fatores clínicos e a constituição das resinas compostas atuais, existem dúvidas quanto ao tempo de irradiação por LED de segunda geração adequado para obtenção das melhores características físicas das RC.

\section{MATERIAL E MÉTODO}

Foram confeccionados 180 discos de resina composta, adquiridas diretamente do revendedor, polimerizados por Luz LED (Bluephase, Ivoclar Vivadent - Schaan, Liechtenstein) com programa de fotopolimerização Soft-Start. Os espécimes foram divididos em três grupos iguais com diferentes protocolos de fotoativação: $10 \mathrm{~s}, 15 \mathrm{~s}$ e $20 \mathrm{~s}$, sendo uma polimerização inicial de $5 \mathrm{~s}$ com potência de 600 $\mathrm{mmW} / \mathrm{cm}^{2}$ e o restante, de acordo com cada subgrupo, de $1.200 \mathrm{mmW} / \mathrm{cm}^{2}$. Destes grupos foram formados 3 subgrupos, dos quais 20 foram confeccionados com resina composta Nano Particulada Filltek Z350 XT (3M ESPE, St. Paul, MN, EUA), 20 com resina composta Nanohíbrida Empress Direct (Ivoclar Vivadent, New Yorc, EUA) e 20 com resina composta microhíbrida Opallis (FGM, Joinville, SC, Brasil), todas de cor A1. De cada subgrupo 10 espécimes foram condicionados em vinho (V) e 10 em água destilada (A) para controle, conforme a Tabela 1.

Tabela 1. Divisão dos grupos e subgrupos segundo o protocolo utilizado e agente corante

\begin{tabular}{|c|c|c|c|}
\hline $\begin{array}{l}\text { Protocolo de } \\
\text { fotopolimerização } \\
\left(\mathbf{m m W} / \mathbf{c m}^{2}\right)\end{array}$ & $\begin{array}{l}\text { G1 } \\
\text { 10 Segundos } \\
5 \mathrm{~s}-600 \\
5 \mathrm{~s}-\mathbf{1 . 2 0 0} \\
\end{array}$ & $\begin{array}{l}\text { G2 } \\
15 \text { Segundos } \\
5 \mathrm{~s}-600 \\
10 \mathrm{~s}-1.200\end{array}$ & $\begin{array}{l}\text { G3 } \\
20 \text { Segundos } \\
5 \text { s - 600 } \\
15 s-1.200\end{array}$ \\
\hline \multirow{5}{*}{ Subgrupo } & Opallis & \multirow{5}{*}{$\begin{array}{l}\text { Opallis } \\
\text { Empress } \\
\text { Direct } \\
\text { Filltek } \\
\text { Z35oXT }\end{array}$} & Opallis \\
\hline & Empress & & Empress \\
\hline & Direct & & Direct \\
\hline & Filltek & & Filltek \\
\hline & Z35oXT & & Z35oXT \\
\hline Agente Corante & A & A & $\mathrm{A}$ \\
\hline
\end{tabular}

Para obtenção dos corpos de prova, foi usada uma matriz de aço com uma perfuração na superfície de $10 \mathrm{~mm}$ de diâmetro e $2 \mathrm{~mm}$ de espessura. Com auxílio de uma espátula (OptraSculpt, Ivoclar Vivadent) as resinas compostas foram inseridas em incremento único. Em seguida, uma matriz de poliéster e uma placa de vidro foram colocadas sobre a resina composta antes da polimerização para uniformizar o polimento superficial dos espécimes.

Um colorímetro (EASYSHADE - Vita, Bad Sackingen, Alemanha) foi usado logo após a confecção dos espécimes e depois de 24 horas, 7, 14 e 21 dias para registrar o $\Delta \mathrm{Lab}$ de cada um dos espécimes. Diversos trabalhos ${ }^{4,18}$ utilizam o sistema de notificação de cor desenvolvido pela CIE (Comission Internationale de l'Eclairage, Comissão Internacional de Iluminação) conhecido como CIELab e que foi escolhido neste estudo. As coordenadas de cor neste sistema tridimensional são $\mathrm{L}=$ coordenada de luminosidade, que é acromática e varia de 0 (preto) a 100 (branco); $a=$ coordenada verde-vermelho (-a: verde, +a: vermelho); e b= coordenada azul-amarelo (-b: azul, $+\mathrm{b}$ : amarelo). As coordenadas a e b são cromáticas. Os valores de $\Delta \mathrm{E}$ foram calculados a partir da fórmula: $\Delta \mathrm{E}=[(\Delta \mathrm{L}) 2+(\Delta \mathrm{a}) 2+(\Delta \mathrm{b}) 2] 1 / 2$.

Após a medição do $\Delta \mathrm{Lab}$ inicial, os espécimes foram acondicionados em placas de Petri e imersos em vinho tinto de $12,5 \%$ volume alcoólico (almadén - cabernet, sauvignon, brasil,) e água destilada, as quais foram a seguir fechadss hermeticamente. 
Os dados obtidos de $\Delta$ Lab inicial (resultante dos valores de 0h e 24h) após 7, 14 e 21 dias foram submetidos à análise estatística descritiva e análise de variância (ANOVA) com nível de significância de 5\%. Quando detectada diferença estatística, os valores foram submetidos ao Teste Duncan para comparações múltiplas.

\section{RESULTADOS}

As médias dos grupos estão descritas na Tabela 2. Na comparação dos deltas entre os momentos, no mesmo indivíduo, foi utilizado o teste $\mathrm{t}$ pareado. $\mathrm{O}$ menor valor encontrado foi $\Delta \mathrm{E}$ 5,49 $\pm 0,37$ para o grupo controle G3 (Empress Direct polimerizado por $20 \mathrm{~s}$ armazenado em água), quando a leitura foi realizada após 24h. Quanto aos resultados dos grupos submersos em vinho, todas as amostras se comportaram de maneira semelhante, ou seja, com o passar do tempo, os valores encontrados eram superiores aos da leitura anterior. A maior alteração ocorreu no grupo G2 (Filteck Z350 XT) ( $\Delta \mathrm{E} 47,30 \pm 2,22$ ) quando mensurado em 21 dias. Houve diferença estatística significante em vários cruzamentos $(\mathrm{p}<0,05)$ (demonstrado pelas letras). Apenas o grupo G1 Opallis, quando comparado entre $7(23,22 \pm 2,15)$ e 14 dias $(23,79 \pm 4,29)$ e o grupo G3 Opallis, quando comparado entre $14(22,90 \pm 4,78)$ e 21 dias $(25,42 \pm 2,02)$, não demonstraram diferença significativa.

Quando foi realizada a comparação do $\Delta \mathrm{E}$ intergrupos (por meio do teste de Duncan) nas diferentes substâncias, percebe-se que também há diferenças entre os grupos, como mostrado na Tabela 3. Quando imersos em água, o grupo G2 Opallis demonstrou a maior média do $\Delta \mathrm{E}$ em 24 h, 7 e 21 dias. O grupo G1 Z350 XT aparece como menor média em todos os momentos.

Tabela 2. Médias do $\Delta \mathrm{E}$ e resultado do teste $\mathrm{t}$ pareado para comparação entre os momentos

\begin{tabular}{|c|c|c|c|c|c|c|}
\hline Grupo & & Substância & $\begin{array}{l}\Delta \mathbf{E} \\
24 \mathbf{H}\end{array}$ & $\begin{array}{l}\Delta \mathbf{E} \\
7 \text { dias }\end{array}$ & $\begin{array}{l}\Delta \mathrm{E} \\
\text { 14dias }\end{array}$ & $\begin{array}{l}\Delta E \\
21 \text { dias }\end{array}$ \\
\hline \multirow{6}{*}{$\begin{array}{c}\text { G1 } \\
(\mathbf{1 0 s})\end{array}$} & \multirow[t]{2}{*}{ Opallis } & Água & $6,59 \mathrm{C}$ & $4,46 \mathrm{~A}$ & $5,41 \mathrm{~B}$ & $5,98 \mathrm{C}$ \\
\hline & & Vinho & $6,32 \mathrm{~A}$ & $23,22 \mathrm{~B}$ & $23,79 \mathrm{~B}$ & $29,22 \mathrm{C}$ \\
\hline & \multirow[t]{2}{*}{ Empress } & Água & $5,61 \mathrm{~B}$ & $3,48 \mathrm{~A}$ & $3,04 \mathrm{~A}$ & $3,28 \mathrm{~A}$ \\
\hline & & Vinho & $6,22 \mathrm{~A}$ & 29,34 B & $34,92 \mathrm{C}$ & $38,98 \mathrm{D}$ \\
\hline & \multirow[t]{2}{*}{ Z350 } & Água & $6,74 \mathrm{C}$ & $2,92 \mathrm{~B}$ & $1,91 \mathrm{~A}$ & $2,59 \mathrm{~B}$ \\
\hline & & Vinho & $6,90 \mathrm{~A}$ & $31,32 \mathrm{~B}$ & $33,27 \mathrm{C}$ & $36,36 \mathrm{D}$ \\
\hline \multirow{6}{*}{$\begin{array}{c}\text { G2 } \\
(15 s)\end{array}$} & \multirow[t]{2}{*}{ Opallis } & Água & $8,41 \mathrm{~B}$ & $10,23 \mathrm{C}$ & $4,81 \mathrm{~A}$ & $11,15 \mathrm{C}$ \\
\hline & & Vinho & $9,33 \mathrm{~A}$ & $24,61 \mathrm{~B}$ & $28,53 \mathrm{C}$ & $33,49 \mathrm{D}$ \\
\hline & \multirow[t]{2}{*}{ Empress } & Água & $9,30 \mathrm{C}$ & $8,17 \mathrm{~B}$ & $2,36 \mathrm{~A}$ & $7,43 \mathrm{~B}$ \\
\hline & & Vinho & $2,31 \mathrm{~A}$ & $26,42 \mathrm{~B}$ & $31,37 \mathrm{C}$ & $37,28 \mathrm{D}$ \\
\hline & \multirow[t]{2}{*}{ Z350 } & Água & $9,32 \mathrm{D}$ & $8,02 \mathrm{C}$ & $2,25 \mathrm{~A}$ & $5,89 \mathrm{~B}$ \\
\hline & & Vinho & $9,29 \mathrm{~A}$ & $36,26 \mathrm{~B}$ & $42,81 \mathrm{C}$ & $47,30 \mathrm{D}$ \\
\hline \multirow{6}{*}{$\begin{array}{c}\text { G3 } \\
(20 s)\end{array}$} & \multirow[t]{2}{*}{ Opallis } & Água & $5,58 \mathrm{~A}$ & $7,08 \mathrm{~B}$ & $9,72 \mathrm{C}$ & $9,55 \mathrm{C}$ \\
\hline & & Vinho & $6,11 \mathrm{~A}$ & $13,19 \mathrm{~B}$ & $22,90 \mathrm{C}$ & $25,42 \mathrm{C}$ \\
\hline & \multirow[t]{2}{*}{ Empress } & Água & $5,49 \mathrm{~B}$ & $3,97 \mathrm{~A}$ & $6,51 \mathrm{C}$ & $4,29 \mathrm{~A}$ \\
\hline & & Vinho & $4,05 \mathrm{~A}$ & $22,88 \mathrm{~B}$ & $35,19 \mathrm{C}$ & $40,39 \mathrm{D}$ \\
\hline & \multirow[t]{2}{*}{ Z350 } & Água & $6,23 \mathrm{~B}$ & $3,93 \mathrm{~A}$ & $5,61 \mathrm{~B}$ & $5,34 \mathrm{~B}$ \\
\hline & & Vinho & $5,39 \mathrm{~A}$ & $23,43 \mathrm{~B}$ & $34,05 \mathrm{C}$ & $40,94 \mathrm{D}$ \\
\hline
\end{tabular}

Em relação aos espécimes submersos em vinho, os resultados obtidos (Tabela 4), demonstram que o grupo G2 Filteck Z350 XT apresenta a maior média do $\Delta \mathrm{E}$ em todos os momentos $(9,29 \pm 0,45$;
$36,26 \pm 3,46 ; 42,81 \pm 1,97 ; 47,30 \pm 2,22)$. O grupo G3 Opallis tem menor média nos momentos 7 $(13,19 \pm 1,26), \quad 14 \quad(22,90 \pm 4,78) \quad$ e 21 dias $(25,42 \pm 2,02)$.

Tabela 3. Comparação vertical intergrupos e resultados do teste de Duncan - Água

\begin{tabular}{|c|c|c|c|c|}
\hline Grupos & $\begin{array}{l}\Delta \mathbf{E} \\
24 H\end{array}$ & $\begin{array}{l}\Delta \mathrm{E} \\
7 \mathrm{dias}\end{array}$ & $\begin{array}{l}\Delta E \\
14 \text { dias }\end{array}$ & $\begin{array}{l}\Delta \mathrm{E} \\
21 \text { dias }\end{array}$ \\
\hline G1 - Opallis & $6,59 \mathrm{~A}$ & $4,46 \mathrm{~B}$ & $5,41 \mathrm{C}$ & $5,98 \mathrm{D}$ \\
\hline G1 - Empress & $5,61 \mathrm{~A}$ & $3,48 \mathrm{~A}, \mathrm{~B}$ & $3,04 \mathrm{~B}$ & $3,28 \mathrm{~A}, \mathrm{~B}$ \\
\hline G1 - Z35o & $6,74 \mathrm{~A}$ & $2,92 \mathrm{~A}$ & $1,91 \mathrm{~A}$ & $2,59 \mathrm{~A}$ \\
\hline G2 - Opallis & $8,41 \mathrm{~B}$ & $10,23 \mathrm{D}$ & $4,81 \mathrm{C}$ & $11,15 \mathrm{G}$ \\
\hline G2 - Empress & $9,30 \mathrm{~B}$ & $8,17 \mathrm{C}$ & $2,36 \mathrm{~A}, \mathrm{~B}$ & $7,43 \mathrm{E}$ \\
\hline G2 - Z350 & $9,32 \mathrm{~B}$ & $8,02 \mathrm{C}$ & $2,25 \mathrm{~A}, \mathrm{~B}$ & $5,89 \mathrm{D}$ \\
\hline G3-Opallis & $5,58 \mathrm{~A}$ & $7,08 \mathrm{C}$ & $9,72 \mathrm{E}$ & $9,55 \mathrm{~F}$ \\
\hline G3 - Empress & $5,49 \mathrm{~A}$ & $3,97 \mathrm{~A}, \mathrm{~B}$ & $6,51 \mathrm{D}$ & $4,29 \mathrm{~B}, \mathrm{C}$ \\
\hline G3 - Z350 & $6,23 \mathrm{~A}$ & 3,93A,B & $5,61 \mathrm{C}$ & $5,34 \mathrm{C}, \mathrm{D}$ \\
\hline p-valor (ANOVA) & 0,000 & 0,000 & 0,000 & 0,000 \\
\hline
\end{tabular}

Tabela 4. Comparação vertical intergrupos e resultados do teste de Duncan - Vinho

\begin{tabular}{|c|c|c|c|c|}
\hline Grupos & $\Delta E 24 H$ & $\Delta \mathrm{E} 7$ dias & $\Delta \mathrm{E} 14$ dias & $\Delta \mathrm{E} 21$ dias \\
\hline G1 - Opallis & 6,32C,D & $23,22 \mathrm{~A}, \mathrm{~B}$ & $23,79 \mathrm{~A}$ & $29,22 \mathrm{~B}$ \\
\hline G1 - Empress & 6,22C,D & 29,34C,D & $34,92 \mathrm{D}$ & $38,98 \mathrm{D}, \mathrm{E}$ \\
\hline G1 - Z350 & $6,90 \mathrm{E}$ & $31,32 \mathrm{D}$ & $33,27 \mathrm{C}, \mathrm{D}$ & $36,36 \mathrm{D}$ \\
\hline G2 - Opallis & $9,33 \mathrm{~F}$ & $24,61 \mathrm{~A}, \mathrm{~B}$ & $28,53 \mathrm{~B}$ & $33,49 \mathrm{C}$ \\
\hline G2 - Empress & $2,31 \mathrm{~A}$ & $26,42 \mathrm{~B}, \mathrm{C}$ & $31,37 \mathrm{~B}, \mathrm{C}$ & $37,28 \mathrm{D}$ \\
\hline G2 - Z350 & $9,29 \mathrm{~F}$ & $36,26 \mathrm{E}$ & $42,81 \mathrm{E}$ & $47,30 \mathrm{~F}$ \\
\hline G3-Opallis & 6,11C,D & $13,19 \mathrm{~A}$ & $22,90 \mathrm{~A}$ & $25,42 \mathrm{~A}$ \\
\hline G3 - Empress & $4,05 \mathrm{~B}$ & $22,88 \mathrm{~A}, \mathrm{~B}$ & $35,19 \mathrm{D}$ & $40,39 \mathrm{E}$ \\
\hline G3 - Z350 & $5,39 \mathrm{C}$ & $23,43 \mathrm{~A}, \mathrm{~B}$ & $34,05 \mathrm{C}, \mathrm{D}$ & $40,94 \mathrm{E}$ \\
\hline p-valor (ANOVA) & 0,000 & 0,000 & 0,000 & 0,000 \\
\hline
\end{tabular}

\section{DISCUSSÃO}

Embora as RC atuais tenham lisura de superfície quase similar ao esmalte, elas sofrem a influência de pigmentos que com o tempo alteram sua coloração inicial ${ }^{18}$. Para melhorar a resistência ao manchamento, as RC devem possuir características intrínsecas adequadas, como composição do material, matriz, conteúdo de carga e componentes de iniciação. Tais características podem ser obtidas por meio de um método de polimerização adequado, que é específico para cada marca comercial da resina composta $^{3,19}$. Pequeno et al. ${ }^{20}$ relatam que a qualidade da polimerização das RC, influencia as propriedades mecânicas do material. Porto et al. ${ }^{11}$ comprovam que materiais mais ricos em fase orgânica apresentam um maior potencial para sofrer manchamento, o que pode ser atribuído ao grau de conversão desses materiais sendo que a presença de monômeros residuais no material resinoso torna-o susceptível à pigmentação por sorção de substâncias externas.

No presente trabalho, todos os grupos imersos em vinho apresentaram pigmentação exacerbada, o que pode ser justificado pelas características inerentes à sua composição. Compósitos que apresentam Bis-GMA em sua composição exibem maior hidrofilicidade e aumento da sorção de água quando comparados àqueles que contêm UDMA, como presente nas resinas Opallis, Empress Direct e Filtek Z350 XT. Quando este último serve de base da matriz resinosa, as resinas 
apresentam maior estabilidade de cor do que outras que apresentam diferentes oligômeros como matrizes. UDMA e também o Bis-EMA criam uma rede polimérica mais rígida e mais hidrófoba, ambos também encontrados nas RC usadas ${ }^{19,21}$. Uma pesquisa demonstrou que a adição, mesmo em pequenas quantidades, de TEGDMA pode aumentar significativamente a sorção de água do material ${ }^{21}$, como é o caso da resina composta Opallis e Filtek Z350 XT utilizadas neste estudo.

A pigmentação aumentada pelo vinho devese ao fato de que além de sua inerente coloração, possui ação fermentativa sobre o compósito devido ao álcool presente em sua composição, sendo este responsável pelo enfraquecimento da estrutura da resina e facilidade de absorção de corantes ${ }^{18}$. O que vai de acordo com os resultados deste estudo, visto que os espécimes condicionados em vinho apresentaram maiores valores de $\Delta \mathrm{E}$, quando comparados aos imersos em água.

Um estudo demonstrou que um compósito nanoparticulado sofre uma mudança de cor maior que um microhíbrido quando submetido ao manchamento com café e vinho ${ }^{22}$. Isto foi verificado nesta pesquisa, uma vez que em todos os protocolos de fotopolimerização o grupo Opallis obteve os menores valores de $\Delta \mathrm{E}$ quando analisados em 7, 14 e 21 dias. Tem-se discutido que o aumento do tamanho das partículas resulta em menor mudança de cor devido a uma diminuição da proporção da matriz orgânica ${ }^{4}$.

Quanto aos tempos de fotopolimerização, Barghi et al. ${ }^{23}$ afirmam que o tempo, a densidade de potência e o comprimento de onda da fonte de luz utilizada para a polimerização do material resinoso são fatores importantes que determinam seu grau de conversão e, consequentemente, a manutenção das propriedades mecânicas e estéticas do material. O LED utilizado neste estudo emite comprimento de onda de 430-490 nm, ou seja, emite luz azul ideal para excitar a CQ. Isto pode explicar porque a resina Opallis, que contém somente a CQ como fotoiniciador, demonstrou menores valores de $\Delta \mathrm{E}$ com um tempo de polimerização aumentado. Entretanto, não se pode observar a mesma relação nas outras RC quando avaliadas em 21 dias. A RC Empress Direct contém Lucirin numa concentração em torno de $2 \%$, que são sensíveis a um comprimento de onda de 380 a $420 \mathrm{~nm}$. Por outro lado, embora a RC Filtek Z350 XT contenha apenas CQ como fotoiniciador seus valores de $\Delta \mathrm{E}$ não diminuíram com o aumento da exposição à luz. Isto sugere que além do tempo de fotopolimerização, outros fatores podem influenciar a pigmentação dos compósitos.

Um estudo realizado em 2012 concluiu que, em relação ao grau de conversão, os dois aparelhos de LED terceira geração utilizados demonstraram um melhor desempenho nos materiais que continham TPO quando comparados aos de segunda geração.
Enquanto a RC que possuía a CQ como fotoiniciador apresentou uma baixa quantidade de conversão quando irradiada pela unidade convencional ${ }^{24}$. Assim, o uso dos LED de segunda e terceira gerações para fotoativação de diferentes RC suscita a necessidade de pesquisas que avaliem a eficácia desta polimerização.

\section{CONCLUSÃO}

Este estudo, dentro de suas limitações, conclui que a imersão em vinho provoca aumento da pigmentação com o passar do tempo. As resinas compostas microhíbridas sofrem menor pigmentação com o passar do tempo comparadas às nanohíbridas ou nanoparticuladas. Resinas compostas com outros fotoiniciadores além da CQ sofrem maior pigmentação ao serem polimerizadas com LED de segunda geração independente do tempo de polimerização e do tempo de armazenamento em substância pigmentante.

\section{REFERÊNCIAS}

1. Matson MR, Matson AMFP. Avaliação do grau de conversão de resinas compostas quando ativadas por luz halógena: método convencional, alta potência e método "soft". Rev Inst Ciênc Saúde. 2006;24(4):307-11.

2. Delaviz Y, Finer Y, Santerre JP. Biodegradation of resin composites and adhesivesby oral bacteria and saliva: A rationale for new material designs that consider the clinical environment and treatment challenges. Dent Mater. 2014;30(1):16-32.

3. Nahsan FPS, Ueda JK, Silva JO, Schimitt VL, Naufel FS, Formighieri LA et al. Estabilidade de cor de resina composta após imersão em café, água e solução de clorexidina. Rev Bras Pesq Saúde. 2009;11(2):13-7.

4. Al Kheraif AA, Qasim SS, Ramakrishnaiah R, Rehman I. Effect of different beverages on the color stability and degree of conversion of nano and microhybrid composites. Dent Mater J. 2013;(32)2:326-31.

5. Ceballos L, Fuentes MV, Tafalla H, Martínez A, Flores J, Rodríguez J. Curing effectiveness of resin composites at different exposure times using LED and halogen units. Med Oral Pathol Oral Cir Bucal 2009;14(1):e51-6.

6. Rahiotis C, Patsouri K, Silikas N, Kakaboura A. Curing efficiency of high- intensity light-emitting diode (LED) devices. J Oral Sci. 2010; 52(2):187-95.

7. Sgarbi SC, Pereira SK, Martins JMH, Oliveira MAC, Mazur RF. Degree of conversion of resin composites light activated by halogen light and led analyzed by ultraviolet spectrometry. Rev Clín Pesq Odontol. 2010;6(3):223-30.

8. Caldarelli PG, Beltrani FC, Santos FA, Shibayama R, Hoepper MG. Manchamento de uma resina composta e sua Relação com o 
efeito da intensidade da luz Emitida por aparelhos fotopolimerizadores de Lâmpada halôgena. Rev Fac Odontol Aracatuba. 2011;(32)2:44-8.

9. Valentini F, Oliveira SGD, Guimarães GZ, Barbosa RPS, Moraes RR. Effect of surface sealant on the color stability of composite resin restorations. Braz Dent J. 2011;22(5):365-68.

10. Freitas ABDA, Gomes HR, Guimarães FBR, Marinho VA, Barros LM. Influência do tipo de aparelho fotopolimerizador na absorção de água por restaurações de resina composta. Rev Inst Ciênc Saúde. 2008;(26)1:65-9.

11.Porto ICCM, Soares LES, Martin AA, Cavalli V, Liporoni PCS. Influence of the photoinitiator system and light photoactivation units on the degree of conversion of dental composites. Braz Oral Res. 2010;24(4):475-81.

12. Pequeno JHP, Araújo GS, Neri JR, Bomfim AGM, Mendonça JS, Santiago SL. Efeito dos protocolos de fotoativação na dureza de uma resina composta nanoparticulada. ROBRAC. 2012;21(56):394-97.

13. Uhl A, Mills RW, Rzanny AE, Jandt KD. Time dependence of composite shrinkage using halogen and LED light curing. Dent Mater. 2005;21(3):278-86.

14.Eick JD, Kotha SP, Chappelow CC, Kilway KV, Giese GJ, Glaros AG et al. Properties of silorane-based dental resins and composites containing a stressreducing monomer. Dent Mater. 2007;23(8):1011-17.

15. Neumann MG, Schmitt CC, Ferreira GC, Correa IC. The initiating radical yields and the efficiency of polymerization for various dental photoinitiators excited by different lightcuring units. Dent Mater. 2006;22(6):576-84.

16. Asmussen E, Peutzfeldt A. Influence of composition on rate of polymerization contraction of light-curing resin composites. Acta Odontol Scand. 2002;60(3):146-50.

17. Silva EH, Albuquerque RC, Lanza LD, Vieira GC, Peixoto RT, Alvim HH et al. Influence of different light sources on the conversion of composite resins. Indian J Dent Res. 2011;22(6):790-94.

18. Souza E, Espíndola M, Diegoli NM, Kuroshima KN. Avaliação da alteração de cor de diferentes compósitos restauradores - influência de corantes usados na cavidade oral - estudo in vitro. RGO. 2006;54(1):43-6.

19. Salvego RN, Dias RPB. Figueiredo JLG. Estabilidade de cor de resinas compostas no processo de manchamento e clareamento, Rev Dental Press Estét. 2013;10(3):54-62.

20. Firoozmandi LM, Balducci I, Araújo MAM. Influência da Fotopolimerização e da Cor da
Resina Composta na Microdureza. Pesq Bras Odontoped Clín Integr. 2009;9(1):37-42.

21. Anfe TEA, Agra CM, Vieira GF. Comparação de duas técnicas de fotoativação na sorção e solubilidade de resinas compostas em solução de etanol. J Biodent Biomater. 2011;1(1):61-7.

22. Villalta P, Lu H, Okte Z, Garcia-Godoy F, Powers JM. Effects of staining and bleaching on color change of dental composite resins. J Prosthet Dent. 2006;95(2):137-42.

23. Barghi N, Berry T, Hatton C. Evaluation intensity output of curing lights in private dental offices. J. am. dent. assoc. 1994;125(7):992-96.

24. Santini A, Miletic V, Swift MD, Bradley M. Degree of conversion and microhardness of TPO-containing resin-based composites cured by polywave and monowave LED units. J Dent. 2012;40(7):577-84.

\section{CONFLITO DE INTERESSES}

Os autores declaram não haver conflitos de interesse.

\section{AUTOR PARA CORRESPONDENCIA}

\section{Luiz Henrique Soares Torres}

luiz-lhst@hotmail.com
Submetido em 11/02/2019

Aceito em 24/05/2019 